\title{
Forskolin Preferentially Dilates the Afferent Arteriole in the Canine Kidney
}

\author{
Toshiaki Tamaki, Kouichi Hasui, Tetsuo Shoji, Yasuharu Aki, Hideyasu Kiyomoto, \\ Hiroshi Iwao and Youichi Abe \\ Department of Pharmacology, Kagawa Medical School, Kagawa 761-07, Japan
}

Received July 14, 1990 Accepred November 8, 1990

\begin{abstract}
Intrarenal infusion of forskolin $(0.2 \mu \mathrm{g} / \mathrm{kg} / \mathrm{min})$ in anesthetized dogs increased renal blood flow (RBF) and glomerular filtration rate (GFR) to the same degree but did not change the mean arterial pressure or heart rate. Forskolin also increased urine flow and urinary sodium excretion. The proportional increases in RBF and GFR may result from a preferential action of forskolin to dilate the afferent arteriole.
\end{abstract}

The role of adenosine $3^{\prime} 5^{\prime}$-cyclic monophosphate (cAMP) as a second messenger of hormone- or drug-induced vasodilation has been investigated. A rise in intracellular cAMP concentration is believed to be responsible for the relaxation of vascular smooth muscle by certain drugs. We previously reported that isoproterenol and glucagon, both stimulators of adenylate cyclase, increased not only renal blood flow (RBF) but also glomerular filtration rate $(\mathrm{GFR})(1,2)$. These results suggest that these renal vasodilators show preferential action for dilating the preglomerular arteriole over the postglomerular arteriole. On the other hand, intrarenal infusion of acetylcholine or bradykinin increase RBF but do not alter GFR $(3,4)$, indicating that acetylcholine and bradykinin dilate both arterioles equally. These findings suggest that the different responses of the afferent and efferent arterioles result from stimulation of a different adenylate cyclase-cAMP system in each arteriole.

Forskolin is a powerful adenylate cyclase stimulator and a good pharmacological tool for investigating the role of cAMP in vasodilation
(5). We have reported that forskolin stimulates the accumulation of cAMP in the isolated afferent arterioles (6). In the present study, we examined the effects of intrarenal infusion of forskolin on renal hemodynamics and renal function and discussed the role of cAMP on the regulation of renal hemodynamics.

Experiments were performed on adult mongrel dogs of both sexes, weighing $9-13 \mathrm{~kg}$, which were maintained on standard laboratory diet for 1 week. The animals were anesthetized with sodium pentobarbital $(30 \mathrm{mg} / \mathrm{kg}$ i.v.) and were given additional doses as required. After tracheotomy, the dogs were mechanically ventilated with room air. Catheters were inserted into the right brachial vein and artery for infusion of saline and for arterial blood sampling. Renal arterial pressure was measured from a catheter inserted into the right femoral artery and advanced into the abdominal aorta just below the left renal artery. The left kidney was exposed through a retroperitoneal flank incision. The kidney was denervated by carefully sectioning all visible nerve fibers associated with the renal vessels. 
RBF was measured with an electromagnetic flowmeter (Nihon Kohden MFV-1200, Japan). Renal arterial pressure, heart rate (HR) and RBF were continuously monitored and recorded on a polygraph (NEC San-ei, Japan). A 23-gauge needle was inserted into the left renal artery proximal to the flow probe for the intrarenal infusion of vehicles and drugs at a rate of $0.19 \mathrm{ml} / \mathrm{min}$. GFR was measured by the creatinine clearance. Another catheter was inserted into the left ureter for urine collection. After completion of surgery, each dog was left undisturbed for $60-90 \mathrm{~min}$ to allow for stabilization of blood pressure, renal blood flow and urine flow.

Urine was collected during three consecutive 10-min control clearance periods. At the mid-point of each period, $5 \mathrm{ml}$ of systemic arterial blood was collected from the right brachial artery. After control periods, forskolin was infused into the renal artery for $30 \mathrm{~min}$ at $0.2 \mu \mathrm{g} / \mathrm{kg} / \mathrm{min}$. Ten minutes after the start of intrarenal infusion, urine and blood samples were collected for two consecutive $10-\mathrm{min}$ clearance periods. Following the 30 -min forskolin infusion, the vehicle was infused into the renal artery again.

Forskolin was dissolved in propylene glycol and diluted in $0.9 \%$ saline. The final concentration of propylene glycol was less than $0.1 \%$. Forskolin was purchased from Sigma Chemicals.

The values presented are means \pm S.E. Statistical analysis was performed by a 2-way analysis of the variance with complete randomized block, and significant differences were determined by the least significance test.

The effects of intrarenal infusion of forskolin on systemic and renal hemodynamics are shown in Fig. 1. Mean arterial pressure (MAP) did not change during the experiment. Heart rate slightly increased during forskolin infusion, but this increment was not statistically significant. RBF increased gradually after the start of intrarenal infusion of forskolin $(0.2$ $\mu \mathrm{g} / \mathrm{kg} / \mathrm{min}$ ), and this increase lasted throughout the forskolin infusion. RBF at $15 \mathrm{~min}$ and 25 min after the start of forskolin infusion was significantly increased by $15 \%$ and $18 \%$, respectively. GFR also increased during forskolin infusion in the same manner as RBF. GFR at $15 \mathrm{~min}$ and $25 \mathrm{~min}$ of forskolin infusion was significantly increased by $12 \%$ and $16 \%$, respectively. The filtration fraction did not change during forskolin infusion (from $0.36 \pm$ 0.04 at control to $0.36 \pm 0.04$ at $25 \mathrm{~min}$ of forskolin infusion). RBF and GFR returned to their control levels $30 \mathrm{~min}$ after cessation of the infusion.

Urine flow and urinary excretion of sodium $\left(\mathrm{U}_{\mathrm{Na}} \mathrm{V}\right)$ increased during forskolin infusion (Table 1). Urine flow and $\mathrm{U}_{\mathrm{Na}} \mathrm{V}$ at the recovery clearance period were not different from the control values. Urinary excretion of potassium increased slightly, but this change was not statistically significant.

These experiments show that intrarenal infusion of forskolin $(0.2 \mu \mathrm{g} / \mathrm{kg} / \mathrm{min})$ increases RBF and GFR to the same degree in dogs. Forskolin has been reported to have a positive inotropic action (5). The systemic hemodyna-

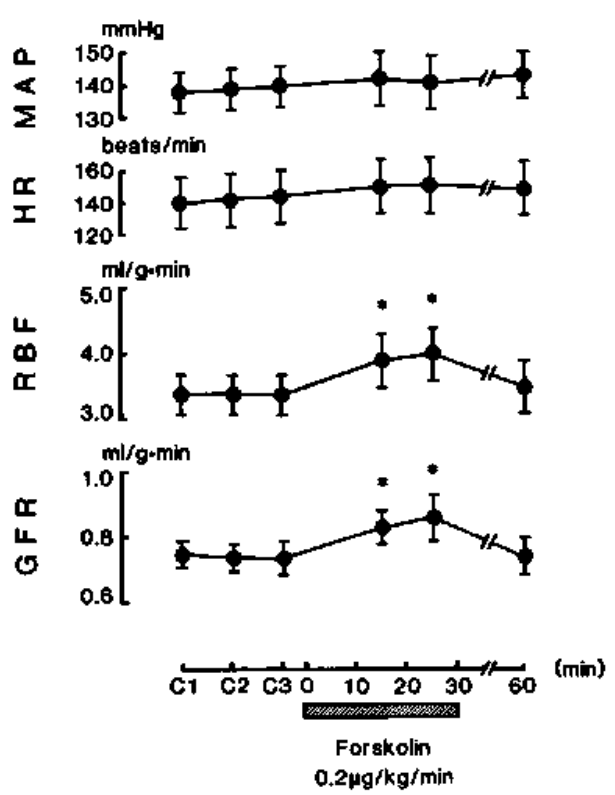

Fig. 1 Effects of intrarenal infusion of forskolin on systemic and renal hemodynamics. MAP: mean arterial pressure, HR: heart rate, RBF: renal blood flow, GFR: glomerular filtration rate, $\mathrm{C}$ : control. ${ }^{*}$ indicates significant difference from $\mathrm{C} 3(P<0.05), n=6$. 
Table 1. Effects of forskolin on urine flow and excretion of electrolytes

\begin{tabular}{llcc}
\hline & $\begin{array}{c}\text { Urine flow } \\
\mu \mathrm{l} / \mathrm{g} \cdot \min \end{array}$ & $\begin{array}{c}\mathrm{U}_{\mathrm{Na}} \mathrm{V} \\
\mu \mathrm{Eq} / \mathrm{g} \cdot \min \end{array}$ & $\begin{array}{c}\mathrm{U}_{\mathrm{K}} \mathrm{V} \\
\mu \mathrm{Eq} / \mathrm{g} \cdot \min \end{array}$ \\
\hline Control & $14.7 \pm 3.4$ & $3.12 \pm 0.76$ & $\mathbf{0 . 4 7} \pm \mathbf{0 . 0 6}$ \\
Forskolin $(0.2 \mu \mathrm{g} / \mathrm{kg} / \mathrm{min})$ & & & \\
$\quad 15 \mathrm{~min}$ & $19.2 \pm 4.2^{*}$ & $4.22 \pm 0.96^{*}$ & $0.64 \pm 0.12$ \\
$25 \mathrm{~min}$ & $20.5 \pm 4.6^{*}$ & $4.47 \pm 0.98^{*}$ & $\mathbf{0 . 6 8} \pm 0.15$ \\
Recovery & $16.2 \pm 3.6$ & $3.59 \pm 0.74$ & $\mathbf{0 . 6 5} \pm 0.12$ \\
\hline
\end{tabular}

Values are means \pm S.E. ${ }^{*}$ indicates significant difference from the control value $(\mathrm{P}<$ $0.05) . \mathrm{n}=6$.

mic changes may influence renal hemodynamics. However, the dose of forskolin that we used did not change systemic blood pressure or heart rate in these experiments. We thought that the intrarenal infusion of forskolin at a rate of $0.2 \mu \mathrm{g} / \mathrm{kg} / \mathrm{min}$ did not influence the systemic hemodynamics. Therefore, the positive inotropic action of forskolin does not appear to be responsible for the increase in RBF. Moreover, we can rule out indirect effects mediated via the renal nerves because we used denervated canine kidneys in this experiment. Thus, forskolin may act on renal resistance vessels directly to increase RBF.

Forskolin is one of the most powerful stimulators of adenylate cyclase. There are many reports that forskolin increases intracellular cAMP content in vascular smooth muscle of various vascular beds. Most forskolin-induced changes have been explained by the accumulation of intracellular cAMP. Many researchers believe that the accumulation of intracellular cAMP in blood vessels results in relaxation. We reported that forskolin increased cAMP accumulation in isolated canine afferent arterioles (6). We propose that the forskolin-induced rise of intracellular cAMP in renal resistance vessels mediates the renal vasodilatory effect of forskolin in these experiments.

The renal circulation is regulated by two resistance vessels, the afferent and efferent arterioles. Acetylcholine has been reported to increase RBF but not to alter GFR because acetylcholine dilates the afferent and efferent arterioles equally. Selective dilation of the afferent arteriole increases the effective filtra- tion pressure in the glomerulus. Therefore, selective dilation of the afferent arteriole may increase not only RBF but also GFR. However, there are other factors which regulate GFR, for example, the mean transcapillary hydrostatic pressure $(\Delta P)$, the mean transcapillary oncotic pressure difference, renal plasma flow (RPF) and glomerular ultrafiltration coefficient (Kf). In acute dog experiments, RPF is not a major determinant of GFR, because vasodilators such as acetylcholine and bradykinin increase RPF but do not affect GFR $(3,4)$. In the present experiment, the filtration fraction in dog kidney during forskolin infusion did not change because of the parallel increases in RBF and GFR. This indicates that if the transcapillary oncotic pressure difference changed, the change was minimal. Ichikawa and Brenner (7) suggested that Kf was decreased by the accumulation of cAMP in the rat. Therefore, we thought that the increase in GFR during forskolin infusion may have resulted from an increase in $\Delta P$. Selective dilation of the afferent arteriole during forskolin infusion may imply a quantitative difference in adenylate cyclase between the afferent and efferent arterioles. We reported that dibutyryl cAMP also increased RBF and GFR (8). These results suggest that an intracellular event(s) after cAMP accumulation may be responsible for the different responses of the two arterioles.

Intrarenal infusion of forskolin increased urine flow even though cAMP is also a second messenger of the antidiuretic hormone (9). We reported that adenylate cyclase stimula- 
tors, isoproterenol and glucagon, and dibutyryl cAMP also increased urine flow $(1,2,8)$. We cannot explain the increased urine flow produced by these agents, but the increase in GFR may have been responsible.

Recently, it was reported that forskolin blocked $\mathrm{K}^{+}$channels independently of adenylate cyclase $(10)$. This property of forskolin may not be responsible for it's renal vasodilatory effect. Most $\mathrm{K}^{+}$channel openers have been reported to relax arterial smooth muscles and to lower blood pressure (11). Hayashi et al. reported that a $\mathrm{K}^{+}$channel opener dilated the renal vasculature (12). Therefore, blocking $\mathrm{K}^{+}$channels may lead to constriction of the resistance vessels.

This study demonstrated that intrarenal infusion of forskolin increased not only RBF but also GFR. These proportional increases of RBF and GFR may result from a preferential action of forskolin to dilate the afferent arteriole. However, further studies are required to clarify the mechanism of the differential action of adenylate cyclase stimulations.

\section{Acknowledgments}

We are grateful to Dr. Ian Reid (San Francisco, CA) for proof-reading this manuscript and C. Kimura and R. Masaki for secretarial services.

\section{REFERENCES}

1 Morimoto, S., Abe, Y. and Yamamoto. K.: Diuretic action of isoproterenol in the dog. Japan. Circ. J. 35, 601-610 (1971)

2 Ueda, J., Nakanishi, H., Miyazaki, M. and Abe, Y.: Effect of glucagon on the renal hemodymanics of dogs. Eur. J. Pharmacol. 41, 209-212 (1977)

3 Stein, J.H., Congbalay, R.C., Karsh, D.L..
Osgood, R.W. and Ferris, T.F.: Effect of bradykinin on proximal tubular sodium reabsorption in the dog: Evidence for functional nephron heterogeneity. J. Clin. Invest. 51, 1709- 1721 (1972)

4 Thomas, C.E., Ott, C.E., Bell, P.D., Knox, F.G. and Navar, L.G.: Glomerular filtration dynamics during renal vasodilation with acetylcholine in the dog. Am. J. Physiol. 244, F606-F611 (1983)

5 de Souza, N.J., Dohadwalla, A.N. and Reden, J.: Forskolin: A labdane diterpenoid with antihypertensive, positive inotropic, platelet aggregation inhibitory, and adenylate cyclase activating properties. Med. Res. Rev. 3, 201 - 219 (1983)

6 Tamaki, T., Hura, C.E. and Kunau, R.T., Jr.: Dopamine stimulates cAMP production in canine afferent arteriole via $\mathrm{DA}_{1}$ receptors. Am. J. Physiol. 256, H626-H629 (1989)

7 Ichikawa, I. and Brenner, B.M.: Evidence for glomerular actions of ADH and dibutyryl cyclic AMP in the rat. Am. J. Physiol. 233, F102-F117 (1977)

8 Okahara, T., Abe, Y. and Yamamoto, K.: Effects of dibutyryl cyclic AMP and propranolol on renin secretion in dogs. Proc. Soc. Exp. Biol. Med. 156, 213-218 (1977)

9 Burg, M.B.: Renal handling of sodium, chloride, water, amino acids, and glucose. In The Kidney, Third Edition, Edited by Bremer, B.M. and Rector, F.C., Jr., Vol. 1, p. 145-175, W.B. Sanders Co., Philadelphia (1986)

10 Hoshi, T., Garber, S.S. and Akdrich, R.W.: Effect of forskolin on voltage-gated $\mathbf{K}^{+}$channels is independent of adenylate cyclase activation. Science 240, $1652-1655$ (1988)

11 Cook, N.S.: The pharmacology of potassium channels and their therapeutic potential. Trends Pharmacol. Sci. 9, 21 - 28 (1988)

12 Hayashi, K., Matsumura, Y., Yoshida, Y., Ohyama, T., Hisaki, K., Suzuki, Y. and Morimoto, S.: Effects of BRL 34915 (Cromakalim) on renal hemodynamics and function in anesthetized dogs. J. Pharmacol. Exp. Ther. 252, 1240-1246 (1990) 